\title{
Improved Ant Colony Optimization in Express Distribution Routing
}

\author{
Tianshi Liu' \\ School of Computer Science, Xi'an Shi you University \\ Xi'an,710065, China \\ E-mail: liutianshiexsyu.edu.cn
}

\section{Shuanshuan Qin , Liumei Zhang}

School of Computer Science, Xi'an Shi you University

Xi'an, 710065, China

E-mail: $370416452 @ q 9 \cdot \mathrm{com}$

Increasingly complex urban traffic conditions often challenge the express services with long delivery path and much more time as consumed. In this paper, a Traffic Impact Factor (TIF) is introduced to model the impact of urban traffic conditions on express services. Based on the TIF, an optimization model is constructed to minimize the delivery distance and the time consumed. In the solution, the objective function is defined by incorporating TIF into the probability transfer formula of the Ant Colony Optimization (ACO), which has thereby improved the update rule of pheromone. The improved ACO is suitable for the optimization of the express delivery path. In the experiment, 30 cities express delivery experimental data are used to compare the distribution distance, the delivery time and the objective function value between the ACO and the improved ACO. The result shows that the improved ACO may reduce the distribution cost related to the distribution distance and the time factor. 


\section{Introduction}

With the rapid development of E-commerce, the distribution of goods has become an important part . To save delivery costs, we need to study the problems of distribution path. There are many ways to solve the problem of vehicle paths, which can be classified into two categories: exact algorithm and heuristic algorithm. The exact algorithm is based on the strict mathematical model. If the problem can be solved, the solution with the exact algorithm is usually better than the heuristic algorithm. But the exact algorithm can not avoid the index explosion problem. According to above all, the exact algorithm can only effectively solve the problem of small-scale vehicle routing, and only apply to a particular situation. For this situation, many scholars have noted heuristic algorithms.

At present, there are many kinds of heuristic algorithms, such as the Simulated Annealing Arithmetic(SAA), the Tabu Search(TS), the Ant Colony Optimization (ACO), the Genetic Algorithm(GA) and so on. In recent years, it is the GA and the TS that have been applied successful to solve the logistics distribution vehicle path problem, but there also exist some disadvantages. For example, the ability of GA to search locally is not strong and not very efficient as a whole when used to find the feasible solution, while the TS is dependent on the initial solution. But the ACO is a method based on the overall optimization. It is not only a population-based simulation evolutionary algorithm, but also a random search algorithm. In addition, it is versatile and robust. In this paper, we firstly set up a model for express delivery costs, in which two factors are considered : delivery distance and delivery time. And then we improve the calculation of transition probability and update pheromone in the ACO. It proves the improved ACO is more effective in optimizing the express delivery routing.

\section{Ant Colony Optimization}

Ant colony optimization is a simulated evolutionary method inspired from the food hunting movement of ant colony in real world. Marco Dorigo proposed the algorithm to solve the traveling salesman problem (TSP). To some extent, the problem of the shortest path selection in express delivery is similar to the TSP. Therefore, this article aims to resolve the shortest path problem in express delivery via the improved ant colony optimization.

At the beginning, the ants explore the surrounding areas randomly, then the pheromone on each path is equal. Ants will choose a path based on the pheromone and heuristic information of each path. When they find that a path which is the shortest, the pheromone is released. Within a certain period o, more ants will take this path, and then more pheromone will be released for the following ants, until all the ants take the shortest path. The transition probability $P_{i j}^{k}(t)$ that the ant $\mathrm{k}$ move from point $\mathrm{I}$ to point $\mathrm{J}$ at $\mathrm{t}$ time shows in equation (2.1):

$$
P_{i j}^{k}(t)=\left(\begin{array}{cc}
\frac{\tau_{i j}^{\alpha}(t) \eta_{i j}^{\beta}(t)}{\sum_{k \in A_{k}} \tau_{i s}^{\alpha}(t) \eta_{i s}^{\beta}(t)} \quad j \in A_{k} \\
0 \quad \text { otherwise }
\end{array}\right)
$$

Where, $\mathrm{k}$ refers to the ant number, $A_{k}$ Refers to the point that the ant has not yet arrived, $\alpha$ refers to the relative importance of pheromones, $\beta$ refers to the relative importance of the heuristic factor, $\tau_{i j}$ refers to an information heuristic factor, also known as pheromone, $\eta_{i j}$ refers to an expected heuristic value usually is the reciprocal of the distance between two points. The formula is shown in Equation(2.2). 


$$
\eta_{i j}=\frac{1}{d_{i j}}
$$

Where, dij represents the distance from point I to point $\mathrm{J}$. As the pheromone of one ant is gradually evaporate over time, the pheromones will be adjusted according to the following Equation (2.3) .

$$
\begin{gathered}
\tau_{i j}(t+n)=(1-\rho) \times \tau_{i j}(t)+\Delta \tau_{i j}(t) \\
\Delta \tau_{i j}(t)=\sum_{k=1}^{m} \Delta \tau_{i j}{ }^{k}(t)
\end{gathered}
$$

Where, $(2.3), \rho(\rho(0,1))$ represents the pheromone evaporation coefficient, and $1-\rho$ is the residual factor. $\Delta \tau_{i j}(t)$ is the pheromone increment in the path $(\mathrm{i}, \mathrm{j})$ of this loop. In equation (2.4), $\Delta \tau_{i j}^{k}(t)$ represents the pheromone of the $\mathrm{k}$ ant in the loop $(\mathrm{i}, \mathrm{j})$ in this cycle. $\mathrm{m}$ is the quantity of ants and $\mathrm{k}$ is the ant number.

\section{The Iimproved Ant Colony Optimization of Eexpress Delivery Route Optimization}

The construction of highway is of utmost significance to the development of transportation industry. Because high speed vehicles on the highway can shorten the running time, and reduce fuel consumption, vehicle consumption and transportation costs. At the same time, due to the highway control mechanism, it also can reduce the traffic accidents, thus increasing safety for divers. Although the express service can use airway transport, most of the express delivery still takes the road transportation. In express delivery process, the distribution of vehicles traveling on different types of road can affect the delivery time obviously. Therefore, this paper aims to optimize the distribution route by reducing distribution distance and time.

\subsection{Traffic Impact Factor and the Influence Coefficient}

In order to depict the route choice of the vehicle with different roads, we propose a traffic impact factor. Although ordinary highways do not charge, most of the roads are poor and the traffic is more crowded. The delivery man should avoid it, considering the time factor. Here we set the ordinary road traffic impact factor as 0.8 , which means that 80 percent of the delivery man is likely to avoid the ordinary highway. On the contrary, Super highways are in good condition and vehicles can travel fast. Therefore, we set the expressway traffic impact factor as 0.2 . In addition, according to the speed of vehicles on ordinary and super highways, we set the average speed of the distribution vehicle of the ordinary highway and super highway respectively as $45 \mathrm{~km} / \mathrm{h}$ and $90 \mathrm{~km} / \mathrm{h}$ respectively. Table 1 below indicates the traffic impact factor and the average speed of the vehicle at different highway grade:

\begin{tabular}{l|l|l}
\hline Highway grade & Ordinary highway & Super highway \\
\hline$\theta$ & 0.8 & 0.2 \\
\hline $\mathrm{V}$ & 45 & 90 \\
\hline
\end{tabular}

Table 1. Impact Factor and the Average Speed of Vehicle in Different Highway Traffic

The traffic impact coefficient is as shown in Equation (3.1):

$$
C=\theta \times d_{i j}
$$

Where, $\theta$ is the traffic impact factor and $\mathrm{D}$ represents the path for the delivery vehicle. $\mathrm{C}$ indicates the traffic impact coefficient when the distribution vehicles choose different grades of highway. The higher $\mathrm{C}$ value indicates the higher liability to avoid the road. 


\subsection{Establishment of Express Delivery Path Planning Objective Function}

In express delivery path planning, we need to consider both the distance of the distribution path and the delivery time. Therefore, we establish the following models by considering the distribution distance and time factor as follows:

$$
\begin{gathered}
F=\min \quad\left(\omega_{1}+\omega_{2} \times \delta \times T\right) \\
D=\min \sum d_{i j} \\
T=\frac{D}{V}=\min \frac{\sum d_{i j}}{v_{i j}}
\end{gathered}
$$

Where, $\mathrm{d}_{\mathrm{ij}}$ stands for the distance between the city I and the city J. D represents the delivery route chosen by the distribution vehicle, and its value is the minimum value of the distance of the delivery route. $\mathrm{V}$ is the average speed of the distribution vehicle. $\mathrm{T}$ indicates the time that the vehicle need to take on different roads, and the value is the minimum value of the travel time of the delivery vehicle. In Formula (3.2), $\omega_{1}$, and $\omega_{2}$ refer to the weight values of D,T. $\delta$ refers to the parameter to ensure $\mathrm{D}$ and $\mathrm{T}$ to have the same grade. $\mathrm{F}$ is a multi-objective decision making model based on the distribution distance and the delivery time, which represents the cost of distribution. According to the above three formulas, we establish the model of express delivery cost to determine the shortest path as quickly as possible.

\subsection{Update Transition Probability}

In the ant colony optimization, the factors that influence the selection of the next city are pheromone and path length. But when considering the delivery time, the traffic impact coefficient also needs to be considered as impacts on the path selection of ants. Thus the probability of movement of ant $\mathrm{k}$ from city $\mathrm{i}$ to city $\mathrm{j}$ can be expressed as shown in equation (3.5) :

$$
P_{i j}^{k}(t)=\left(\begin{array}{ll}
\frac{\tau_{i j}^{\alpha}(t) \eta_{i j}^{\beta}(t) \mu_{i j}^{\gamma}(t)}{\sum_{k \in \text { allowed }_{k}} \tau_{i s}^{\alpha}(t) \eta_{i s}^{\beta}(t) \mu_{i s}^{\gamma}(t)} & j \in \text { allowed }_{k} \\
0 & \text { otherwise }
\end{array}\right)
$$

When compared to the probability transfer formula of ant colony algorithm, Equation (3.5) is added with parameter $\mu_{i j}(t)$ and $\gamma . \mu_{i j}(t)$ represents the traffic decision degree of path (i-j )in $\mathrm{t}$ time. $\gamma$ reflects the influence of $\mu_{i j}(t)$ on the probability of ant transfer. After adding the parameters $\mu_{i j}(t)$ and $\gamma$, the transfer probability of ants is determined by three factors: the pheromone $\tau_{i j}$, the expectation heuristic factor $\eta_{i j}$ and the traffic decision $\mu_{i j}(t)$. The $\mu_{i j}(t)$. is defined as follows:

$$
\mu_{i j}(t)=\frac{1}{C_{i j}+1}
$$

Where, $C_{i j}$ represents the traffic impact factor when the distribution vehicle chooses different grades of highways at the path I-J , and it is inversely proportional to $\mu_{i j}(t)$. The bigger the value of $C_{i j}$, the more the distribution vehicle avoids the road, and the smaller the traffic decision degree.

\subsection{Pheromone Update Strategy}


Since the goal of the proposed model is to find the optimal combination of distribution distance and time, the pheromone left on the path $(i, j)$ after the end of the ant $k$ loop in the algorithm process is expressed by the following equation:

$$
\Delta \tau_{i j}^{k}(t)=\left(\begin{array}{cc}
\frac{Q}{F_{k}} & i, j \in F_{k} \\
0 & \text { otherwise }
\end{array}\right)
$$

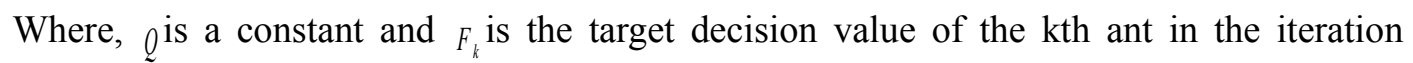
process, and also represents the cost of delivery.

\section{Experimental Analysis and Comparison}

Experiment is conducted by using Matlab to simulate express delivery then validates the proposed model. Assuming that the shipment company needs to ship the goods to 30 cities including Lhasa, Beijing, Shanghai, Tianjin, etc, the cargo vehicle is required to pass through 30 cities and each city only once, and seek the shortest path to return to the company in the shortest time.Relative coordinates of each city are shown in Table 2 below as derived from the part of the relative coordinates in solving TSP problem of the 144 cities in China.

\begin{tabular}{|c|c|c|c|c|c|c|c|}
\hline \multirow{2}{*}{$\begin{array}{l}\text { Serial } \\
\text { number }\end{array}$} & \multirow{2}{*}{ The name of the city } & \multicolumn{2}{|c|}{$\begin{array}{l}\text { Urban geography } \\
\text { coordinates }\end{array}$} & \multirow{2}{*}{$\begin{array}{l}\text { Serial } \\
\text { number }\end{array}$} & \multirow{2}{*}{$\begin{array}{l}\text { The name of the } \\
\text { city }\end{array}$} & \multicolumn{2}{|c|}{$\begin{array}{l}\text { Urban geography } \\
\text { coordinates }\end{array}$} \\
\hline & & $\mathrm{X}$-axis & $\mathrm{Y}$-axis & & & $\mathrm{X}$-axis & $Y$-axis \\
\hline 1 & Lhasa & 1304 & 3212 & 2 & Beijing & 3639 & 1315 \\
\hline 3 & Shanghai & 4177 & 2244 & 4 & Tianjin & 3712 & 1399 \\
\hline 5 & Shijiazhuang & 3488 & 1535 & 6 & Taiyuan & 3326 & 1556 \\
\hline 7 & Hohhot & 3238 & 1229 & 8 & Shenyang & 4196 & 1044 \\
\hline 9 & Changchun & 4312 & 790 & 10 & Harbin & 4386 & 570 \\
\hline 11 & Xi'an & 3077 & 1970 & 12 & Lanzhou & 2562 & 1756 \\
\hline 13 & Yinchuan & 2788 & 1491 & 14 & Xining & 2381 & 1676 \\
\hline 15 & Urumchi & 1332 & 695 & 16 & Ji'nan & 3715 & 1678 \\
\hline 17 & Nanjing & 3918 & 2179 & 18 & Hangzhou & 4061 & 2370 \\
\hline 19 & Hefei & 3780 & 2212 & 20 & Nanchang & 3676 & 2578 \\
\hline 21 & Fuzhou & 4029 & 2838 & 22 & Zhengzhou & 3429 & 1908 \\
\hline 23 & Wuhan & 3507 & 2376 & 24 & Changsha & 3494 & 3201 \\
\hline 25 & Guangzhou & 3439 & 3201 & 26 & Nanning & 2935 & 3240 \\
\hline 27 & Haikou & 3140 & 3557 & 28 & Chengdu & 2545 & 2357 \\
\hline 29 & Guiyang & 2778 & 2826 & 30 & Kunming & 2370 & 2975 \\
\hline
\end{tabular}

Table 2: Relative Coordinates of Each City

The ant colony optimization and the improved ant colony optimization are used to calculate the city position in this paper. In addition, we use the actual situation of the highway between cities, such as the speed of vehicle, the distance, the time and the distribution path to calculate. In the improved algorithm, after a lot of experiments, the parameters are set as: $\mathrm{m}=30, \alpha=1, \beta=2, \gamma=3, \rho=0.5, \omega 1=0.5, \omega 2=0.5, \delta=100, \mathrm{Q}=1500, \mathrm{NC} \_\max =100$. The results of the two 

algorithms obtain the optimal path, the shortest distance and the shortest time respectively. Figure (1) and (2) show the optimal path of ant colony optimization and improved ant colony optimization respectively. The left side of Figure (3) and (4) show the shortest distance (indicated by the red line) and the average distance (indicated by the blue line) when the optimal path is selected. Whereas the right side shows the shortest time (indicated by red line) and the average time (indicated by the blue line) when the optimal path is selected.

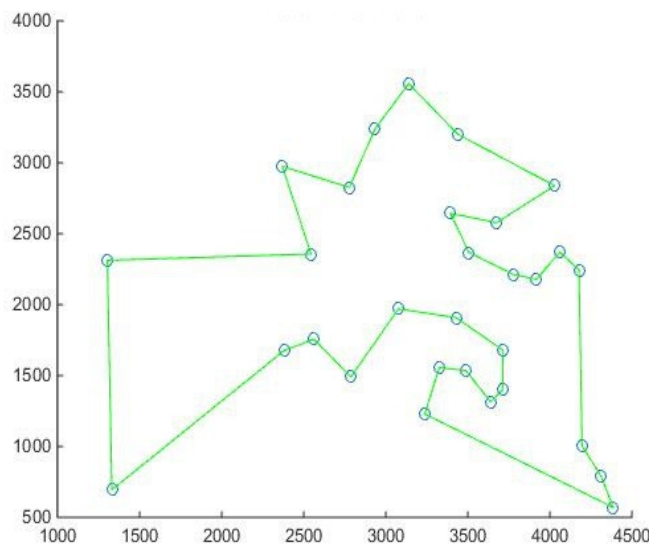

Figure 1: Optimal Path of ACO
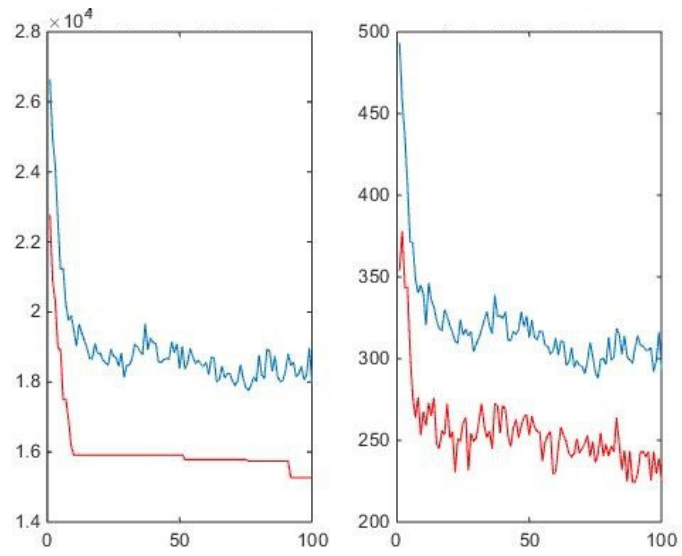

Figure 3: The Shortest Distance and the Shortest Time of ACO

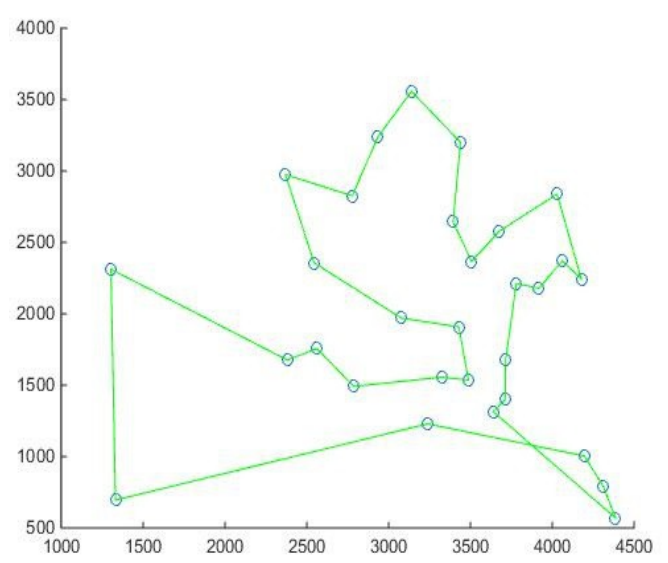

Figure 2: Optimal Path of the Improved ACO
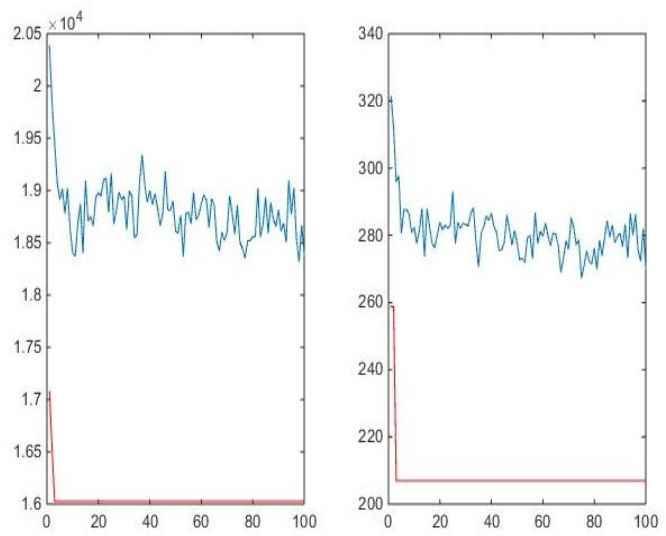

Figure 4:The Shortest Distance and the Shortest Time of the Improved ACO

The result of ant colony optimization is:

(1) Optimal path: 1-15-14-12-13-11-22-16-4-2-5-6-7-10-9-8-3-18-17-19-23-24-2021-25-27-26-29-30-28

(2) The shortest distance: $1.5270 \mathrm{e}+04 \mathrm{~km}$

(3) The least time: $251.0239 \mathrm{~h}$

(4) Target function value: $4.0372 \mathrm{e}+04$

The result of improved ant colony optimization is:

(1) The optimal path: 2-4-16-19-17-18-3-21-20-23-24-25-27-26-29-30-28-11-22-56-13-12-14-1-15-7-8-9-10

(2) The shortest distance: $1.6017 \mathrm{e}+04 \mathrm{~km}$

(3) The least time: $206.9832 \mathrm{~h}$

(4) Target function value: $3.6715 \mathrm{e}+04$ 
Experimental results show that although the ant colony optimization implements the optimal delivery path on express delivery issues, it only considers the delivery distance factor. Furthermore, the delivery time is relatively long and the objective function value is comparatively large. But the improved ant colony optimization takes into account the two factors including the distribution distance and the time. Although the delivery path is not optimal, the delivery time and the objective function value are obviously smaller than both in the ant colony algorithm, in this sense, the results of the improved algorithm are relatively better when compared with the unimproved algorithm. As a result, we can draw such a conclusion that using the improved ant colony algorithm to solve the problem of express delivery route optimization has obvious effect in case of the minimum distribution cost, when considering the distribution distance and the time .

\section{Conclusion}

In this paper, an express delivery cost model is proposed on the base of analyzing the actual situation of express delivery and taking into account the distance factor and time factor of express delivery. Then, according to the requirement of model solving, we improve the transition probability and pheromone by updating rule of the ant colony optimization. Finally according to the actual traffic data, the two algorithms are compared. And the experimental results show that the improved algorithm is more practical and effective in the actual express delivery. But the article only considers the distribution of vehicles to choose different levels of road, which affects the delivery time. If we consider the different levels of road freight, the courier delivery costs will be further reduced, and the optimal distribution path will be better. This is the focus of the further researches.

\section{References}

[1]Duan Haibin. Ant colony optimization and its application [C]. Beijing: Duan Haibin, 2015

[2]Liu lan. Communication problem and its ant colony optimization improvement under traffic constraints and soft time window conditions [J]. Logistics Technology, 2016, 35 (9): 91-96

[3]Murrary A, Gerrard R.A. Capacitated service and regional constraints in location-allocation modeling[J].Location Science,1997,5(2):103-118.

[4]Pan Xiaomeng. Ant colony optimization optimization and path planning problem application research [J]. Science and Technology Bulletin, 2016, 32 (6): 99-103

[5]Tang Chenliang. Optimization of public travel path based on ant colony optimization algorithm and taxi GPS data [J]. Journal of China Highway, 2011, 24 (2): 89-96

[6]Tang Liang. Path Planning Method Based on Improved Ant Colony optimization [J]. Journal of University of Science and Technology of China, 2009, 39 (9): 980-995

[7]Vaidyanathan J, Anthony R.A simulated annealing methodology to distribution network design and management $[\mathrm{J}]$.European $\mathrm{J}$ of opeational research,2003,144,(3):629-645

[8] Wu Huafeng. Ant colony optimization based on natural selection strategy to solve TSP problem [J]. Journal of Communications, 2013, 32 (4): 165-170

[9]Yang Baoshi. Feed routing optimization and customer service impact-based ant colony optimization [J]. Logistics Engineering and Management, 2013, 35 (9): 81-83

[10]Yang Congping, Evaluation of Express Logistics Distribution Routing Based on Ant Colony optimization [J]. Logistics Engineering and Management, 2014, 36 (4): 65-68 